\title{
A PASSIVITY-BASED APPROACH TO DEPLOYMENT IN MULTI-AGENT NETWORKS
}

\author{
Heath LeBlanc, Emeka Eyisi, Nicholas Kottenstette, Xenofon Koutsoukos, Janos Sztipanovits \\ Institute for Software Integrated Systems (ISIS), Vanderbilt University, 2015 Terrace Place, Nashville, TN 37203, USA \\ heath.j.leblanc@vanderbilt.edu,emeka.p.eyisi@vanderbilt.edu,nkottens@isis.vanderbilt.edu, \\ xenofon.koutsoukos@vanderbilt.edu,janos.sztipanovits@vanderbilt.edu
}

Keywords: Passivity, compositionality, deployment, overlay network, input-output stability, distributed protocol.

\begin{abstract}
Surveillance and convoy tracking applications often require groups of networked agents for redundancy and better coverage. An important goal upon deployment is to establish a formation around a target. Although there exist distributed algorithms using only local communication that achieve this goal, they typically ignore destabilizing effects resulting from implementation uncertainties, such as network delays and data loss. This paper resolves these issues by introducing a discrete-time distributed design framework that uses a compositional, passivity-based approach to ensure $l_{2}^{m}$-stability regardless of overlay network topology, in the presence of network delays and data loss. For the restricted case of a uniform node degree in the overlay network topology, the paper shows that asymptotic formation establishment is achieved. Finally, simulations of velocity-limited unmanned air vehicles (UAVs) are presented that demonstrate the robustness of the network architecture to network delays and data loss.
\end{abstract}

\section{INTRODUCTION}

Modern surveillance and convoy tracking applications often require deploying groups of unmanned aerial vehicles (UAVs). The benefit of using multiple UAVs is redundancy, which reduces the likelihood of missing interesting events on the ground, in the presence of obstructions caused by nonuniform terrain, vegetation, or man-made structures. Further, the additional UAVs provide greater breadth of coverage. A central task for such multi-agent systems is to establish a formation around an area of interest. For example, an $n$-gon with a target as its center, at the appropriate radius, may simultaneously provide significant redundancy and breadth of coverage.

Performing coordinated tasks in multi-agent systems using only local information has been studied extensively over the past decade (Olfati-Saber et al., 2007), (Ren et al., 2005), (Olfati-Saber, 2006). Typically, in group coordination the desired formation emerges from the design of the control law. In (Fax and Murray, 2004), the so-called information filter is used for formation stability of LTI systems. For coordination of nonlinear systems, contraction theory with wave variable communication (Wang and Slotine, 2006), explicit design of Lyapunov vector fields (Lawrence et al., 2008), and passivity (Arcak, 2007), (Ihle et al., 2007), (Bai et al., 2008), (Igarashi et al., 2008), have been used successfully.

Much of the above work - especially the passivitybased methods - has considered continuous-time systems; however, for implementation discrete-time design is needed. In addition, implementation uncertainties such as network delays and data loss must be taken into consideration. This paper focuses on decoupling the control design and discrete-time implementation by using a passivity-based framework inspired by work in telemanipulation (Chopra et al., 2008), port-Hamiltonian systems (Stramigioli et al., 2005), and network control (Kottenstette et al., 2009).

The unifying concept in the aforementioned work is the scattering formalism, which has traditionally been applied to power variables (effort and flow) while closing the loop on velocity. In this work, the scattering formalism is used abstractly (without the physical interpretation) to close the loop on position.

The contributions of this paper are three-fold. First, we introduce a compositional network control 
system (NCS) design approach that guarantees passivity of the networked system. Secondly, we show that the coupled multi-agent network is $l_{2}^{m}$-stable for any bidirectional overlay network with asymmetric delays whenever the input-output mapping of each agent is strictly-output passive. The stability result holds for packet-switched networks using easily enforced constraints. Thirdly, for the single-input, single-output (SISO) case, we perform steady-state analysis and we show that the multi-agent network can establish an $n$-gon upon deployment. Finally, we provide simulations using Simulink/TrueTime to illustrate the approach for controlling velocity-limited quadrotor UAVs. Simulink is a graphical user environment (GUI) used for the modeling, simulation, and analysis of dynamical systems (MathWorks, 2008). TrueTime extends Simulink with platform related modeling concepts (i.e., networks, clocks, schedulers) and supports simulation of networked and embedded control systems with implementation effects (Ohlin et al., 2007).

The rest of the paper is organized as follows: Section 2 provides the formal problem statement and other preliminaries. The distributed NCS design framework is introduced in Section 3. The main theoretical results are detailed in Section 4. Section 5 presents simulations in Simulink/TrueTime illustrating our results. Finally, Section 6 provides concluding remarks and future work.

\section{PRELIMINARIES}

Consider the problem of $n$ agents establishing a formation around a target in $\mathbb{R}^{2}$. Assume a global inertial coordinate system and suppose the starting positions of the agents are arbitrary. The goal is to establish an $n$-gon, where the $n$ agents tend to the coordinates of the vertices asymptotically. Formally, we assign a vertex $v_{i}$ of the $n$-gon to agent $i$, with position $x_{i}(k)$, $i=1,2, \ldots, n$. Then we require

$$
\lim _{k \rightarrow \infty}\left\|x_{i}(k)-v_{i}\right\|_{2}=0 .
$$

We consider a network of $n$ interacting agents with communication topology described by a connected undirected graph, $G=(V, E)$, where $V=\{1,2, \ldots, n\}$ describes the agents and $E=\{(i, j)\}$ models the bidirectional communication. Additionally, each bidirectional link may have asymmetric, time-varying delays. The delays are denoted $d_{i j}(k)$ for link $(i, j) \in E$.

For the purpose of analysis, it is useful to introduce the adjacency matrix, $A=\left[a_{i j}\right]$, associated with graph $G$ (Godsil and Royle, 2001). For an undirected graph, the adjacency matrix is a symmetric matrix (i.e., $A=A^{\top}$ ), and is mathematically defined by

$$
a_{i j}= \begin{cases}1 & (i, j) \in E \\ 0 & (i, j) \notin E .\end{cases}
$$

Additionally, we define the set of neighbors, $N_{i}$, of a node $i$ as those nodes which send messages to $i$, given by $N_{i}=\left\{j \in V \mid a_{j i} \neq 0\right\}$. Finally, we denote the number of neighbors by $\left|N_{i}\right|=n_{i}$.

The agents communicate and process signals in the extended $l_{2}$-space of functions that map $\mathbb{N} \cup\{0\}$ to $\mathbb{R}^{m}$, denoted $l_{2 e}^{m}$, which are mapped onto $l_{2}^{m}$ by the truncation operator defined by

$$
(f)_{N}= \begin{cases}f(k) & 0 \leq k \leq N-1 \\ 0 & \text { otherwise }\end{cases}
$$

Further, for all $f, g \in l_{2 e}^{m}$ define

$$
\langle f, g\rangle_{N} \triangleq \sum_{k=0}^{N-1} f^{\top}(k) g(k) .
$$

We use definitions for $l_{2}^{m}$-stability and passivity for discrete-time systems, which are analogous to the continuous-time counterparts in (van der Schaft, 1999):

Definition 1 Given a discrete-time system defined by its input-output mapping, $G: l_{2 e}^{m} \rightarrow l_{2 e}^{m}$, the discretetime system is $l_{2}^{m}$-stable if

$$
u \in l_{2}^{m} \Longrightarrow G(u) \in l_{2}^{m} .
$$

Definition 2 Let $G: l_{2 e}^{m} \rightarrow l_{2 e}^{m}$. Then, for all $u \in l_{2 e}^{m}$ :

1. $G$ is passive if there exists some constant $\beta \in \mathbb{R}$ (called the bias) such that

$$
\langle G(u), u\rangle_{N} \geq-\beta, \quad \forall N \in \mathbb{N} ;
$$

2. $G$ is strictly output passive if there exists some constants $\beta \in \mathbb{R}$ and $\varepsilon>0$ such that

$$
\langle G(u), u\rangle_{N} \geq \varepsilon\left\|(G(u))_{N}\right\|_{2}^{2}-\beta, \quad \forall N \in \mathbb{N} .
$$

We assume a synchronous network, with period $T{ }^{1}$ Further, each agent shares information only locally (no global shared resources). However, the desired setpoints are calculated prior to deployment. Finally, the agents begin execution at time index $k=0$.

\footnotetext{
${ }^{1} \mathrm{We}$ assume the agents use a clock synchronization algorithm prior to deployment to ensure this assumption holds.
} 


\section{NCS DESIGN}

This section details the distributed network control system (NCS) design. The objective is to provide a passive-by-construction, discrete-time multi-agent network. In general, the overlay network is bidirectional with asymmetric delays. For simplicity, consider the three node network shown in Figure 1. Each node represents a UAV, with each edge modeling the communication between UAVs. Realistically, each link in the network is subject to delay imposed by packet handling and transmission delays. This is modeled by the time-varying delays (e.g., $d_{i j}(k)$ ), shown in Figure 1. The $u$ and $v$ variables in the figure are power wave variables, which are described in Section 3.2.

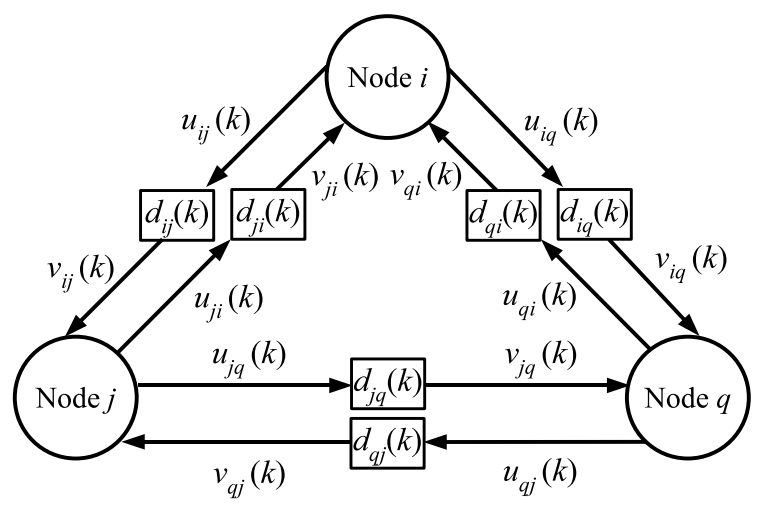

Figure 1: A three node network with time-varying delays in the communication links.

\subsection{Agent Model}

The agent model is shown in Figure 2. Each agent $i$ receives an input reference, $r_{i}$, which influences the output, $y_{i}$, of the agent through the system mapping, $H_{i} . H_{i}$ describes a compensated plant, and is required to be strictly output passive. The variables $x_{i}$ and $y_{i}$ are transformed into the wave domain through the scattering transformation. The node's wave variables $u_{i i}$ and $v_{i i}$ are coupled to other nodes through a power junction, $P J_{i}$, which allows two or more systems to be connected in a passivity-preserving manner (Kottenstette et al., 2009). The scattering transformation and power junction are crucial to ensuring passivity of the networked system and will be described in the next section.

For simplicity, we model the UAVs with a point mass along two dimensions. We denote the point mass system, $H_{p}: f_{I} \rightarrow y_{I}$, in which $f_{I} \in \mathbb{R}^{2}$ is the inertial control force and $y_{I} \in \mathbb{R}^{2}$ is the inertial position as depicted in Figure 3. The equations of motion

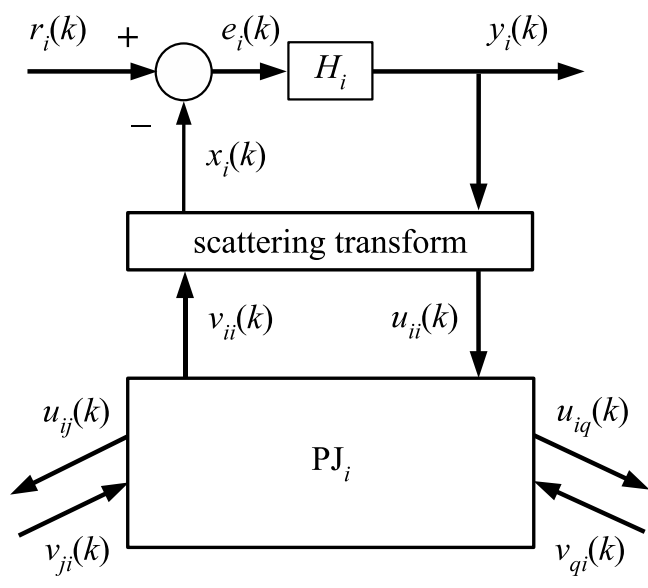

Figure 2: Node architecture.

are

$$
\begin{gathered}
\dot{y}_{I}(t)=v_{I}(t) \\
\dot{M}_{I}(t)=f_{I}(t) .
\end{gathered}
$$

Using the point mass model for each agent $i$, we design an inertial position control system, which we denote $H_{I}: e_{i} \rightarrow y_{I}$, shown in Figure 3 . The inner loop gain of the compensator is $\omega_{c} M\left(\omega_{c}>0\right)$ and the outer loop gain $\frac{\omega_{c}}{2}$. The overall equation of motion

$$
\ddot{y}_{I}=-\omega_{c} \dot{y}_{I}-\frac{\omega_{c}^{2}}{2}\left(y_{I}-e_{i}\right)=-2 \zeta \omega_{n} \dot{y}_{I}-\omega_{n}^{2}\left(y_{I}-e_{i}\right)
$$

clearly indicates a stable second order system with natural frequency $\omega_{n}=\frac{\omega_{c}}{\sqrt{2}}$ and damping coefficient $\zeta=\frac{1}{\sqrt{2}}$, where $y_{I}=e_{i}$ at steady state. It can be shown that the inertial position control system is inside the sector $[a, 1]$, where $a=-\frac{1}{2(1+\sqrt{2})}$ (Zames, 1966), (Kottenstette and Porter, 2009). Therefore, the system $H_{I}: e_{i} \rightarrow y_{I}$ is not strictly output passive; however, by adding a high-pass filter in parallel, the system may be rendered strictly output passive, as depicted in Figure 4 (with $c=2$ ). Since $e_{i}=y_{i}=y_{I}$ at steady state, the inertial position of the system may be directly controlled. This model is discretized using a bilinear-like transform, called the inner-product equivalent sample and hold (IPESH) transform, which preserves the conic properties of the system (Kottenstette et al., 2009).

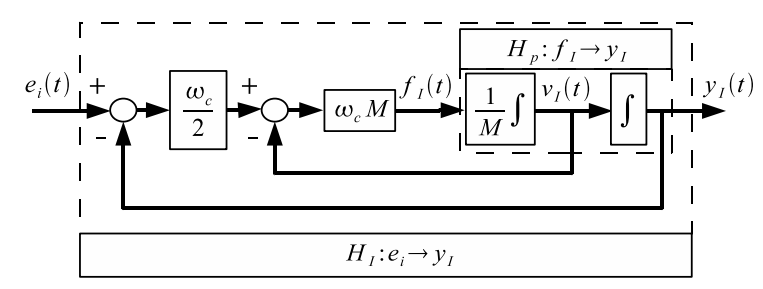

Figure 3: Inertial position control system. 


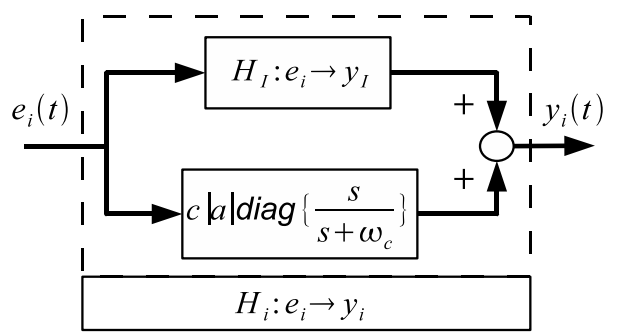

Figure 4: Strictly output passive inertial position control system.

\subsection{Network Model}

In distributed control applications the information transmitted across the network has inherent physical meaning. It is well known that transforming these physical variables into the wave domain can preserve passivity and stability for a single bidirectional connection (Chopra et al., 2008) and for star networks (Kottenstette et al., 2009). In this paper, we extend these approaches to distributed networks with arbitrary overlay topology. The network model is distributed in the sense that all nodes in the network communicate only locally.

We formally define the scattering transformation as follows. For each $i \in V$, the scattering transformation produces power waves $u_{i i}(k)$ and $v_{i i}(k)$ defined by

$$
\begin{aligned}
& u_{i i}(k)=\frac{1}{\sqrt{2 b_{i}}}\left(b_{i} y_{i}(k)+x_{i}(k)\right), \\
& v_{i i}(k)=\frac{1}{\sqrt{2 b_{i}}}\left(b_{i} y_{i}(k)-x_{i}(k)\right) .
\end{aligned}
$$

This definition is similar to the one in (Niemeyer and Slotine, 2004), with the force and velocity variables replaced with $x_{i}$ and $y_{i}$. In general, we place no restriction on the physical meaning of $x_{i}$ and $y_{i}$; however, for our UAV model, $x_{i}$ and $y_{i}$ denote position. The scattering transformation is treated as a mathematical definition, with the characteristic impedance, $b_{i}$, having appropriate units for physical consistency.

Next, we define the power junction, which allows two or more systems to be connected in the wave domain in a passivity-preserving manner.

Definition 3 Fix $m, p \in \mathbb{N}, p \geq 2$. Then, a power junction is a function $f: l_{2 e}^{m p} \rightarrow l_{2 e}^{m p}$, which satisfies for all $\xi \in l_{2 e}^{m p}$ and all $k \in \mathbb{Z}^{+}$the inequality

$$
\xi^{\top}(k) \xi(k) \geq f(\xi(k))^{\top} f(\xi(k)) .
$$

The vector $\xi(k)$ in the definition of the power junction is formed by concatenating the $p$ inputs in $l_{2 e}^{m}$ into a single $m p$-dimensional column vector. For analyzing our network model, it is useful to pair the $p$ inputs to their corresponding outputs in the output column vector, $f(\xi(k))$, and partition the set of pairs into two disjoint sets $S_{\text {in }}$ and $S_{\text {out }}$. These sets denote the net flow of power into and out of the power junction, respectively. Formally, for $i \in S_{\text {in }}$ and $o \in S_{\text {out }}$, let $u_{i}, v_{o} \in l_{2 e}^{m}$ denote the inputs and $v_{i}, u_{o} \in l_{2 e}^{m}$ denote the outputs of the power junction. Then (10) may be rewritten as

$$
\begin{aligned}
& \sum_{i \in S_{\text {in }}} u_{i}^{\top}(k) u_{i}(k)-v_{i}^{\top}(k) v_{i}(k) \geq \\
& \sum_{o \in S_{\text {out }}} u_{o}^{\top}(k) u_{o}(k)-v_{o}^{\top}(k) v_{o}(k) .
\end{aligned}
$$

We implement each node's power junction as a linear set of equations. Specifically, we use the following equations. For each $i \in V, j \in N_{i}$, and $k \in \mathbb{Z}^{+}$, the outgoing waves are computed as

$$
\begin{gathered}
u_{i j}(k)=\frac{1}{\sqrt{n_{i}}} u_{i i}(k), \\
v_{i i}(k)=\frac{1}{\sqrt{n_{i}}} \sum_{j \in N_{i}} v_{j i}(k) .
\end{gathered}
$$

Although the functional form of the power junction is not constrained to be linear, these equations simplify the steady state analysis and exhibit a local averaging behavior in regular networks. This can be seen as follows. Consider the wave variables that influence the power junction at a given node $i$, shown in Figure 5, and suppose $n_{i}=n_{j} \equiv \eta, \forall i, j \in V$ (i.e., a regular network). Then, for each $j \in N_{i}, v_{j i}(k)=$ $u_{j i}\left(k-d_{j i}(k)\right)$. Thus, an expression for $v_{i i}(k)$ is given by

$$
\begin{aligned}
v_{i i}(k) & =\frac{1}{\sqrt{\eta}} \sum_{j \in N_{i}} v_{j i}(k) \\
& =\frac{1}{\sqrt{\eta}} \sum_{j \in N_{i}} u_{j i}\left(k-d_{j i}(k)\right) \\
& =\frac{1}{\sqrt{\eta}} \sum_{j \in N_{i}} \frac{1}{\sqrt{\eta}} u_{j j}\left(k-d_{j i}(k)\right) \\
& =\frac{1}{\eta} \sum_{j \in N_{i}} u_{j j}\left(k-d_{j i}(k)\right) .
\end{aligned}
$$

Therefore, in regular networks, the input wave variable, $v_{i i}(k)$, is the average of its neighbors' delayed output wave variables, $u_{j j}\left(k-d_{j i}(k)\right), j \in N_{i}$.

Due to the presence of delays and data loss some (or all) of the $v_{j i}(k)$ may not be received at time $k$, in which case $v_{j i}(k) \triangleq 0$. Handling delayed and dropped packets as null packets satisfies the synchronous assumption and preserves passivity (Chopra et al., 2008). Before proceeding to describe the constraints on delayed and lost data, we prove our claim that the implementation given by (12) satisfies the definition of a power junction.

Lemma 1 The implementation defined by (12) satisfies the power junction constraint.

Proof: From the remarks following the power junction definition, it is sufficient to show that (12) 


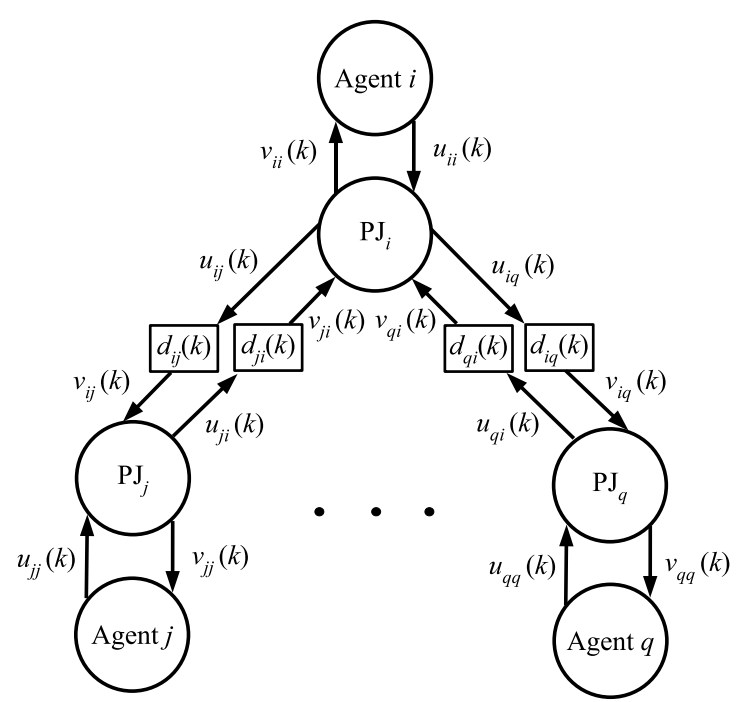

Figure 5: The neighbors of a node, showing the wave variables influencing the node through the power junction.

satisfy (11). Clearly, a sufficient condition for satisfying (11) is to enforce the following constraints for each component $l=1,2, \ldots, m$,

$$
\begin{aligned}
& \sum_{j \in N_{i}} u_{i j_{l}}^{2}(k) \leq u_{i i_{l}}^{2}(k), \\
& v_{i i_{l}}^{2}(k) \leq \sum_{j \in N_{i}} v_{j i_{l}}^{2}(k),
\end{aligned}
$$

where $S_{\text {in }}=\{i i\}$ and $S_{\text {out }}=\left\{i j \mid j \in N_{i}\right\}$. To show that (13a) is satisfied, we use (12a), which yields

$$
\sum_{j \in N_{i}} u_{i j_{l}}^{2}(k)=\sum_{j \in N_{i}} \frac{1}{n_{i}} u_{i i_{l}}^{2}(k)=u_{i i_{l}}^{2}(k) .
$$

For (13b) we combine (12b) with the CauchySchwartz inequality to get

$$
v_{i i_{l}}^{2}(k)=\frac{1}{n_{i}}\left(\sum_{j \in N_{i}} v_{j i_{l}}(k)\right)^{2} \leq \sum_{j \in N_{i}} v_{j i_{l}}^{2}(k) .
$$

Finally, we constrain the network model by preventing retransmission of data for each agent. Also, as mentioned above, whenever receiver's buffers are empty, we process null packets. Based on these assumptions, each channel $(i, j) \in E$ satisfies the following inequality regardless of time-varying delays and data loss (Chopra et al., 2008),

$$
\left\|\left(v_{i j}\right)_{N}\right\|_{2}^{2} \leq\left\|\left(u_{i j}\right)_{N}\right\|_{2}^{2} \text {, holds } \forall N \in \mathbb{N} \text {. }
$$

This inequality states that each channel, viewed as the input-output mapping shown in Figure 6, is passive.

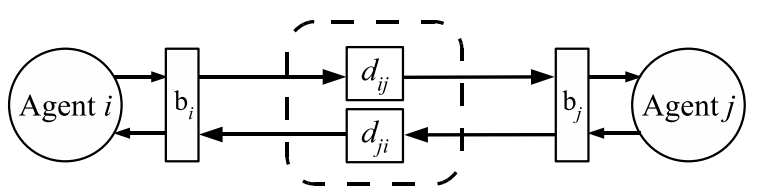

Figure 6: A point-to-point connection using the scattering formalism to ensure passivity of the bidirectional connection subject to asymmetric time-varying delays, shown inside the dashed box.

\section{ANALYSIS}

\subsection{Passivity of the Networked System}

In this section we first prove that the network model is passive and then show that the input-output mapping describing the networked system is strictly output passive. Figure 7 shows the passive network. The following lemma proves that the portion inside the dashed box of Figure 7 is passive.

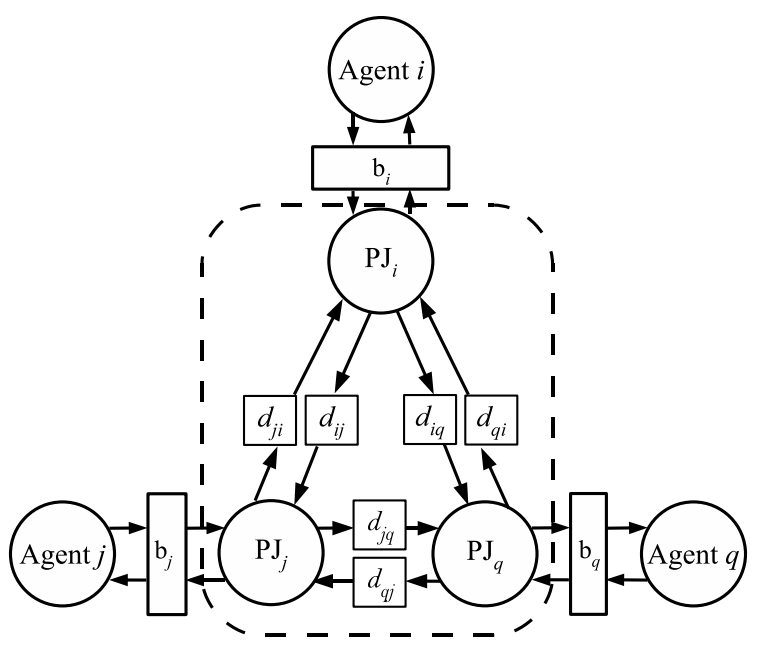

Figure 7: A three node network illustrating the passive network, shown inside the dashed box.

Lemma 2 Consider a network of $n$ interacting $d y$ namic systems constrained to the design framework described in Section 3. Then, the global energy constraint

$$
\sum_{i=1}^{n}\left\{\left\|\left(u_{i i}\right)_{N}\right\|_{2}^{2}-\left\|\left(v_{i i}\right)_{N}\right\|_{2}^{2}\right\} \geq 0
$$

is satisfied for all $N \in \mathbb{N}$, regardless of time-varying delays and data loss.

Proof: Sum the power constraints (11) of each node $i$, with $S_{i n_{i}}=\{i i\}$ and $S_{\text {out }_{i}}=\left\{i j \mid j \in N_{i}\right\}$, from time $k=0$ to $k=N-1$ and sum the resulting inequalities over all nodes (rearranging the terms in the sums ap- 
propriately). Then, invoke (14) to obtain

$$
\begin{aligned}
\sum_{i=1}^{n}\left\{\left\|\left(u_{i i}\right)_{N}\right\|_{2}^{2}-\right. & \left.\left\|\left(v_{i i}\right)_{N}\right\|_{2}^{2}\right\} \\
& \geq \sum_{i=1}^{n} \sum_{j \in N_{i}} a_{i j}\left\{\left\|\left(u_{i j}\right)_{N}\right\|_{2}^{2}-\left\|\left(v_{i j}\right)_{N}\right\|_{2}^{2}\right\} \\
& \geq 0 .
\end{aligned}
$$

The energy constraint of (15) also implies that collectively, the mapping from the $x_{i}$ to the $y_{i}, i=$ $1, \ldots, n$, is passive (see Figure 2). To show this, consider the following power constraint, which may easily be derived from (9a) and (9b)

$$
\frac{1}{2}\left(u_{i i}^{\top}(k) u_{i i}(k)-v_{i i}^{\top}(k) v_{i i}(k)\right)=y_{i}^{\top}(k) x_{i}(k) .
$$

Substitute (16) into (15) to obtain

$$
\sum_{i=1}^{n}\left\langle y_{i}, x_{i}\right\rangle_{N} \geq 0
$$

Define $x(k)$ and $y(k)$ as the $n m \times 1$ column vectors formed by concatenating the $x_{i}(k)$ and $y_{i}(k)$, respectively, of each node. Then, it follows that

$$
\langle y, x\rangle_{N} \geq 0,
$$

which satisfies the definition of passivity (6), with $\beta=$ 0 .

We conclude the section by proving that the entire networked system (e.g., the three node system in Figure 7) is strictly output passive for arbitrary network topologies.

Theorem 1 Consider a network of $n$ interacting $d y$ namic systems constrained to the design framework described in Section 3. Define $r(k)$ and $y(k)$ as the $n m \times 1$ column vectors formed by concatenating the $r_{i}(k)$ and $y_{i}(k)$, respectively, of each node. Finally, define the input-output mapping $H: l_{2 e}^{n m} \rightarrow l_{2 e}^{n m}$ such that $H(r(k))=y(k)$. Then, $H$ is strictly output passive.

Proof: Since each $H_{i}$ is strictly output passive, there exists $\varepsilon_{i}>0$ and $\beta_{i}$, for all $i \in V$, such that

$$
\left\langle y_{i}, e_{i}\right\rangle_{N} \geq \varepsilon_{i}\left\|\left(y_{i}\right)_{N}\right\|_{2}^{2}-\beta_{i} \text {. }
$$

Making the substitution, $x_{i}(k)=r_{i}(k)-e_{i}(k)$ into (17) and using the linearity of the inner-product, gives

$$
\sum_{i=1}^{n}\left\langle y_{i}, r_{i}\right\rangle_{N} \geq \sum_{i=1}^{n}\left\langle y_{i}, e_{i}\right\rangle_{N} .
$$

Substituting (18) into (19) yields

$$
\sum_{i=1}^{n}\left\langle y_{i}, r_{i}\right\rangle_{N} \geq \varepsilon \sum_{i=1}^{n}\left\|\left(y_{i}\right)_{N}\right\|_{2}^{2}-\beta,
$$

where $\varepsilon=\min _{i}\left\{\varepsilon_{i}\right\}$ and $\beta=\sum_{i=1}^{n} \beta_{i}$. Finally, we rewrite (20) as

$$
\langle y, r\rangle_{N} \geq \varepsilon\left\|(y)_{N}\right\|_{2}^{2}-\beta .
$$

\subsection{Stability}

The previous result shows that the networked system defined by the mapping $H$ is strictly output passive. It then follows that $H$ is $l_{2}^{m}$-stable.

Theorem 2 The mapping $H(r(k))=y(k)$ defined in Theorem 1 is $l_{2}^{m}$-stable.

Proof: We begin with the notion of finite $l_{2}^{m}$-gain. The map $G$ has finite $l_{2}^{m}$-gain if there exists finite constants $\gamma, \beta$ such that for all $N \in \mathbb{N}$

$$
\left\|(G(u))_{N}\right\|_{2} \leq \gamma\left\|(u)_{N}\right\|_{2}+\beta, \quad \forall u \in l_{2 e}^{m} .
$$

It is well known in continuous-time (van der Schaft, 1999) and has been shown for discrete-time (Kottenstette and Antsaklis, 2007) that a sufficient condition for a system to have finite $l_{2}^{m}$-gain is for the system to be strictly output passive. Therefore, by Theorem 1, $H$ has finite $l_{2}^{m}$-gain.

Now suppose $u \in l_{2}^{m}$ (i.e., $\left.\|u\|_{2}<\infty\right)$. Then take $N \rightarrow \infty$ in (22). This leads to

$$
\|G(u)\|_{2} \leq \gamma\|u\|_{2}+\beta<\infty, \quad \forall u \in l_{2}^{m} .
$$

Therefore, $H(u) \in l_{2}^{m}$. By Definition $1, H$ is $l_{2}^{m}$-stable.

From the proof of Theorem 2, we see that any system that is strictly output passive is necessarily $l_{2}^{m}$-stable. Therefore, each agent described by $H_{i}$ is inherently stable. The benefit of the passivity-based network framework is that it ensures that interactions caused by the network do not destabilize the networked multi-agent system. This result holds even in the presence of time-varying delays and data loss (under the assumptions outlined in Section 3.2) because the passivity results hold. Moreover, the networked multi-agent system will remain stable regardless of network topology.

\subsection{Steady-State Analysis}

To analyze the behavior of the coupled multi-agent system, we consider the system at steady-state. In order to do this, we assume that each strictly output system, $H_{i}$, admits a steady-state solution whenever a constant input is applied. With this assumption, there exists a steady-state solution for the multi-agent system (provided there is no data loss), since the rest of the networked system is linear. For simplicity, we assume the system is SISO. If the degrees of freedom of the system are decoupled, this result may be applied to MIMO systems.

Theorem 3 Consider a network of $n$ interacting SISO agents designed using the framework described 
in Section 3 and ignore time delays and data loss. Assume the inputs, $r_{i}$, reach steady-state and consider the outputs, $y_{i}$, as $k \rightarrow \infty$. If $H_{i}$ at each node $i$ has steady-state gain $g_{i}$, then the steady-state output of node $i$ is given by

$$
y_{i}=\frac{g_{i}}{b_{i} g_{i}+1}\left[r_{i}+\frac{\sqrt{2 b_{i}}}{\sqrt{\bar{n}_{i}}} \sum_{j \in N_{i}} \frac{1}{\sqrt{2 b_{j} n_{j}}}\left[\frac{b_{j} g_{j}-1}{g_{j}} y_{j}+r_{j}\right]\right]
$$

Proof: Since time delays and data loss are ignored, we drop the time index. Using the relation $e_{i}=r_{i}-x_{i}$ and replacing $H_{i}$ with $g_{i}$, the input-output relation $y_{i}=H_{i}\left(e_{i}\right)$ may be written as

$$
y_{i}=g_{i}\left(r_{i}-x_{i}\right) .
$$

Next, substituting (24) into (9b) and solving for $x_{i}$ yields

$$
x_{i}=\frac{-\sqrt{2 b_{i}}}{b_{i} g_{i}+1} v_{i i}+\frac{b_{i} g_{i}}{b_{i} g_{i}+1} r_{i} .
$$

Substituting (25) into (24) and reducing gives us

$$
y_{i}=\frac{g_{i}}{b_{i} g_{i}+1} r_{i}+\frac{\sqrt{2 b_{i}} g_{i}}{b_{i} g_{i}+1} v_{i i} .
$$

Combining $v_{j i}=u_{j i}$ with (12a) at node $j$ (roles of $j$ and $i$ are reversed), produces

$$
v_{j i}=\frac{1}{\sqrt{n_{j}}} u_{j j} .
$$

Substituting this into (12b) for node $i$ yields

$$
v_{i i}=\frac{1}{\sqrt{n_{i}}} \sum_{j \in N_{i}} \frac{1}{\sqrt{n_{j}}} u_{j j} .
$$

Now, solving (24) at node $j$ for $x_{j}$ and substituting into (9a) at node $j$ produces

$$
u_{j j}=\frac{1}{\sqrt{2 b_{j}}}\left(\frac{b_{j} g_{j}-1}{g_{j}} y_{j}+r_{j}\right)
$$

Substitute (28) into (27) to get

$$
v_{i i}=\frac{1}{\sqrt{n_{i}}} \sum_{j \in N_{i}} \frac{1}{\sqrt{2 b_{j} n_{j}}}\left(\frac{b_{j} g_{j}-1}{g_{j}} y_{j}+r_{j}\right)
$$

Finally, substitute (29) into (26) to obtain (23).

Theorem 3 provides a system of $n$ equations describing the system asymptotically (as $k \rightarrow \infty$ ). The system of equations described by (23) are clearly coupled and depend on the overlay network structure. For the case of a regular topology, the following corollary characterizes the system of equations and provides the means to precalculate the reference inputs to asymptotically achieve a desired setpoint. For the two-dimensional agent model described in Section 3.1 the two degrees of freedom are decoupled, so we use this corollary to establish an $n$-gon around the target, as described in Section 2.
Corollary 1 Consider a network of $n$ SISO agents with a regular overlay network topology (i.e., $n_{i}=$ $n_{j} \equiv \eta \forall i, j \in V$ ). If all of the systems $H_{i}$ have identical steady-state gain $g$ and each scattering transformation has the same impedance $b$, the system of steady-state equations may be written as

$$
y=\frac{g}{b g+1}\left(r+\frac{1}{\eta} A\left[\frac{b g-1}{g} y+r\right]\right),
$$

where $y$ and $r$ are defined in Theorem 1 and $A$ is the adjacency matrix of the regular overlay network topology. Assuming the inverse of $(\eta I+A)$ exists, we may solve this equation for $r$ to obtain

$$
r=\frac{1}{g}(\eta I+A)^{-1}((b g+1) \eta I-(b g-1) A) y .
$$

\section{SIMULATIONS}

The experimental setup involves a network of eight UAVs that communicate in a regular overlay network topology, each with degree $\eta=4$, and a synchronous sampling period of $T=0.01$ seconds. Each UAV moves in the plane, influenced by its own input and the wave variables received from its neighbors. We model the UAVs as described in Section 3.1, so that each has a steady state gain, $g=1$, and characteristic impedance, $b=1$. The dynamics of the velocity limited UAVs are implemented using Simulink models while TrueTime is used to simulate the network dynamics and communication between neighboring UAVs. The network protocol used is IEEE $802.11 \mathrm{~b}$, with a speed of $11 \mathrm{Mbps}$.

\subsection{Evaluation}

We present five scenarios to demonstrate our design framework.

Experiment 1: Nominal Case. In this experiment, delays and data loss are ignored. Figure 8a shows a plot of the eight UAVs enclosing the target at the origin, within a radius of $100 \mathrm{~m}$. The data points show the evolution of the UAVs from their initial positions to their final positions. The UAVs cooperatively enclose the target and each agent is $100 \mathrm{~m}$ away from the target, thus achieving the desired goal.

Figure $8 \mathrm{~b}$ shows the $\mathrm{x}$-positions of four agents (UAVs 1, 3, 5, and 8) over a period of 200 seconds. The desired $\mathrm{x}$-positions for the four agents are $100 \mathrm{~m}$, $0 \mathrm{~m}, 0 \mathrm{~m}$ and $-100 \mathrm{~m}$, respectively. These values correspond to the desired configuration and are achieved in about 160 seconds.

Figure 9 shows the average and maximum errors of all the UAVs' positions relative to the desired configuration. From the figure, the average and maximum error reach the value of zero after 160 seconds 
which corresponds to the time the UAVs achieve the desired configuration.

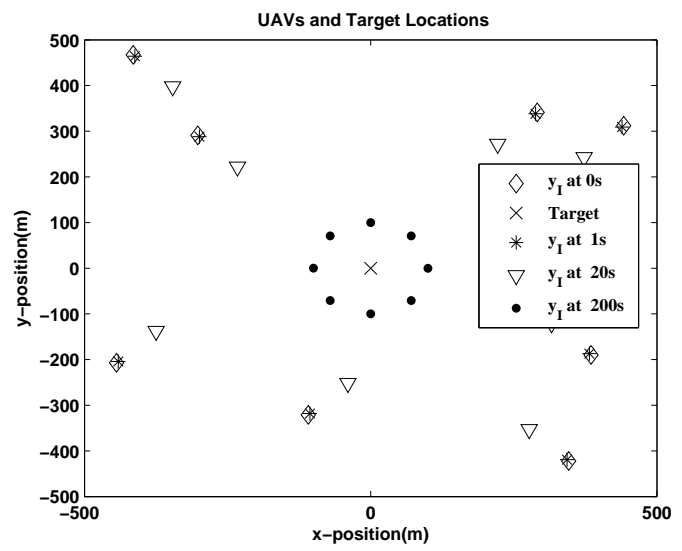

(a) UAVs and target positions.

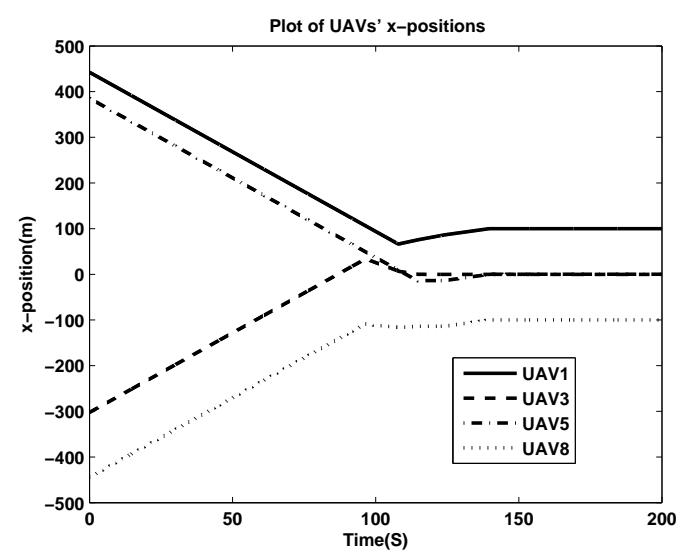

(b) Plot of UAVs' x-position over time.

Figure 8: Network of UAVs (nominal case).

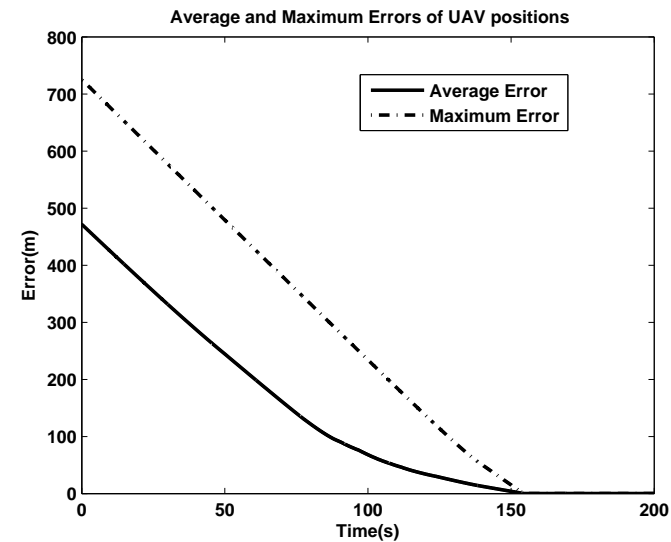

Figure 9: Average and maximum errors of UAVs' positions (nominal case).

Experiment 2: Nonuniform Constant Time Delays. This experiment demonstrates the robustness of

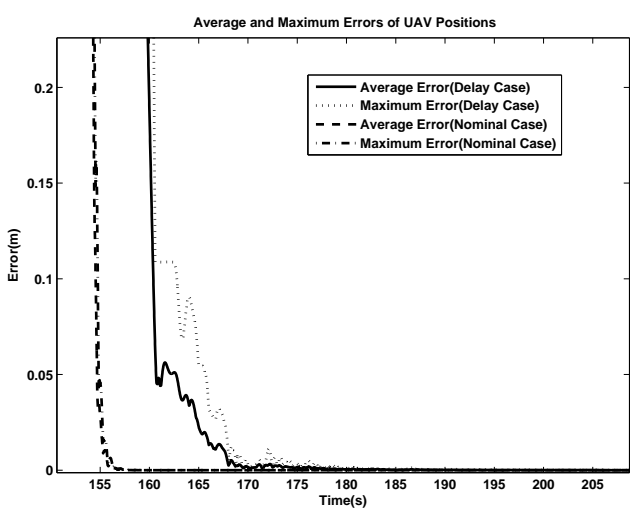

Figure 10: Average and maximum errors of UAVs' positions (nonuniform constant delay in all communication channels).

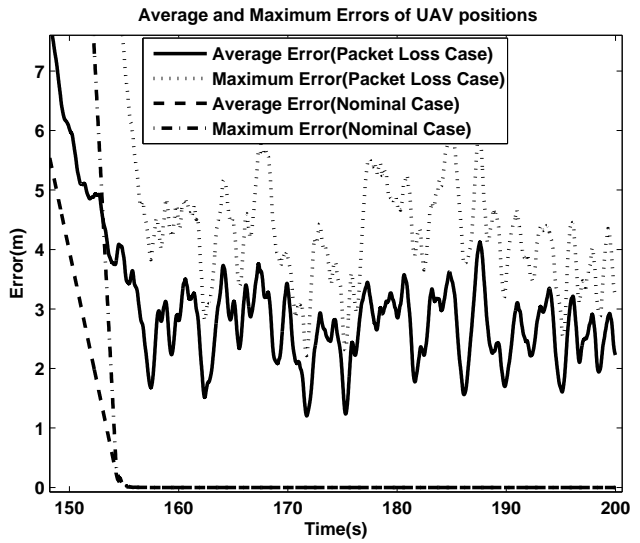

Figure 11: Average and maximum errors of UAVs' positions (ten percent probability of packet loss).

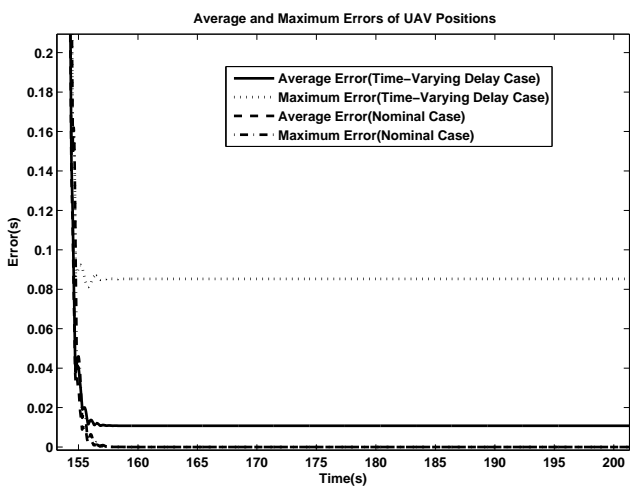

Figure 12: Average and maximum errors of UAVs' positions (time-varying delay case).

the distributed network of UAVs to nonuniform constant delays. We introduce nonuniform time delays, between 1 to 10 seconds, in all the communication channels of the network. Figure 10 shows the average and maximum errors, comparing the nominal case to the case with nonuniform constant delays. From the figure, the average and maximum errors for the delay 


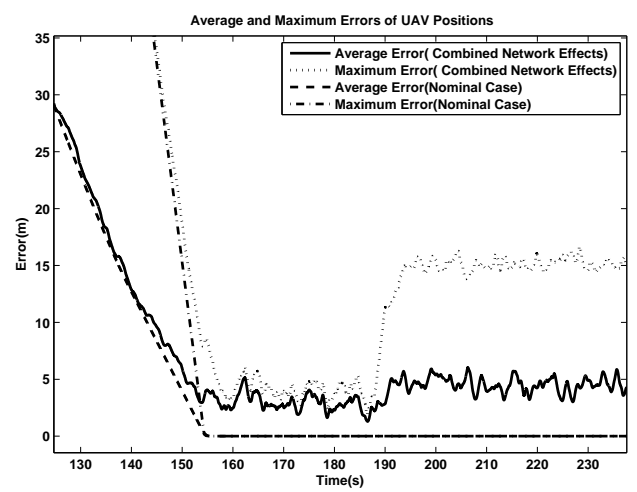

Figure 13: Average and maximum errors of UAVs' positions (time-varying delay and packet loss case).

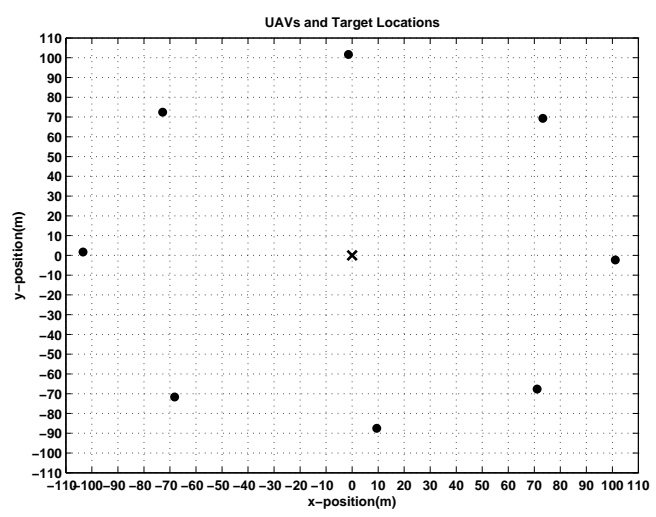

Figure 14: UAVs and target positions (time-varying delay and packet loss case).

case reach the value of zero after 200 seconds, taking about 40 seconds more time than in the nominal case to reach the desired configuration. The presence of time delays in the network does not prevent the agents from reaching the desired configuration; however, the time delays increase the time it takes to reach the desired configuration.

Experiment 3: Data Dropouts. This experiment demonstrates the effect of packet loss on the behavior of the UAVs. A probabilistic model is used to implement the loss of packets in the channels. For our studies, we simulate the case of a ten percent probability of packet loss. Figure 11 shows the average and maximum errors, comparing the nominal case to the case of ten percent probability of packet loss. The plot shows that even with ten percent packet loss, the UAVs still manage to come very close to the desired configuration, demonstrating the resilience of the network. Due to the packet loss, the UAVs will never reach a steady state; however, the UAVs' positions end up within a maximum error of 6 meters and an average error of 4 meters of the desired configuration.

Experiment 4: Time-Varying Delays. This experiment demonstrates the effect of time-varying de- lays on the behavior of the UAVs. To simulate the case of time-varying delays, we incorporate a disturbance node in the network. The sampling period of the disturbance node is set to a value of 0.05 seconds, and the disturbance node floods the network with disturbance packets based on a Bernoulli process with parameter $d$. The disturbance node samples a uniformly distributed random variable $X[k] \in[0,1]$ every 0.05 seconds. If $X[k]>d$, a disturbance packet is forced on the network. Figure 12 shows the average and maximum errors, comparing the nominal case to the time-varying delay case, with $d=0.5$. The plot shows that in the presence of time-varying delays, the UAVs remain stable and settle within a maximum error of 0.09 meters and an average error of 0.02 meters from the desired configuration.

Experiment 5: Combined Network effects. The experiment demonstrates the combined effects of ten percent packet loss and time-varying delays on the behavior of the UAVs. This experiment studies the combined network effects of time-varying delays and data loss in order to simulate the real world dynamics of the network. Again, the time-varying delays are introduced through the disturbance node with $d=0.5$. Figure 13 shows the average and maximum errors, comparing the nominal case to the case with the combined network effects. The figure shows that the average and maximum errors increase slightly after appearing to settle near 5 meters. This occurs because one of the UAVs actually moves interior to the circle around the target, shown in Figure 14. The UAV directly below the target is approximately 15 meters away from its desired location, and causes the maximum error seen in Figure 13.

\section{CONCLUSIONS}

Discrete-time implementation of networked multiagent systems introduces significant challenges caused by effects such as network delays and data loss. This paper proposes a passive-by-construction distributed network control design framework that ensures $l_{2}^{m}$-stability in the presence of these network effects. Using steady-state analysis, we show how to control the agents in the multi-agent network in order to establish an $n$-gon upon deployment. Simulations supporting the theoretical results are presented on the Simulink/TrueTime platform. In future work, we plan to extend the design framework to achieve other group oriented tasks such as output synchronization, formation control, and rendezvous. We will also extend the work to formations in $\mathbb{R}^{3}$. 


\section{ACKNOWLEDGEMENTS}

This work is supported in part by the National Science Foundation (NSF CCF-0820088), the U.S. Army Research Office (ARO W911NF-10-1-0005), the U.S. Air Force Office of Scientific Research (MURI FA9550-06-0312), the U.S Army Research Laboratory (ARL W911NF-087-2-0004), and LockheedMartin. The views and conclusions contained herein are those of the authors and should not be interpreted as necessarily representing the official policies or endorsements, either expressed or implied, of the U.S. Government.

\section{REFERENCES}

Arcak, M. (2007). Passivity as a design tool for group coordination. IEEE Transactions on Automatic Control, 52(8):1380-1390.

Bai, H., Arcak, M., and Wen, J. T. (2008). Rigid body attitude coordination without inertial frame information. Automatica, 44(12):3170 - 3175.

Chopra, N., Berestesky, P., and Spong, M. (2008). Bilateral teleoperation over unreliable communication networks. IEEE Transactions on Control Systems Technology, 16(2):304-313.

Fax, J. A. and Murray, R. M. (2004). Information flow and cooperative control of vehicle formations. IEEE Transactions on Automatic Control, 49(9):1465 1476.

Godsil, C. and Royle, G. (2001). Algebraic Graph Theory. Springer-Verlag New York, Inc.

Igarashi, Y., Hatanaka, T., Fujita, M., and Spong, M. (2008). Passivity-based output synchronization in se(3). In American Control Conference, pages 723-728.

Ihle, I.-A. F., Arcak, M., and Fossen, T. I. (2007). Passivitybased designs for synchronized path-following. Automatica, 43(9):1508 - 1518 .

Kottenstette, N. and Antsaklis, P. (2007). Stable digital control networks for continuous passive plants subject to delays and data dropouts. 46th IEEE Conference on Decision and Control, pages 4433-4440.

Kottenstette, N., Hall, J., Koutsoukos, X., Antsaklis, P., and Sztipanovits, J. (2009). Digital control of multiple discrete passive plants over networks. International Journal of Systems, Control and Communications (IJSCC): Special Issue on Progress in Networked Control Systems. To Appear.

Kottenstette, N. and Porter, J. (2009). Digital Passive Attitude and Altitude Control Schemes for Quadrotor Aircraft. 7th International Conference on Control and Automation.

Lawrence, D. A., Frew, E. W., and Pisano, W. J. (2008). Lyapunov vector fields for autonomous uav flight control. AIAA Journal of Guidance, Control, and Dynamics, 31(5):1220-1229.
MathWorks, I. T. (2008). Simulink. Dynamic System Simulation for MATLAB, Version 7.1.

Niemeyer, G. and Slotine, J.-J. E. (2004). Telemanipulation with time delays. International Journal of Robotics Research, 23(9):873 - 890.

Ohlin, M., Henriksson, D., and Cervin, A. (2007). TrueTime 1.5 Reference Manual. Dept. of Automatic Control, Lund University, Sweden. http://www.control.lth.se/truetime/.

Olfati-Saber, R. (2006). Flocking for multi-agent dynamic systems: algorithms and theory. IEEE Transactions on Automatic Control, 51(3):401-420.

Olfati-Saber, R., Fax, J. A., and Murray, R. M. (2007). Consensus and cooperation in networked multi-agent systems. Proceedings of the IEEE, 95(1):215-233.

Ren, W., Beard, R., and Atkins, E. (2005). A survey of consensus problems in multi-agent coordination. In Proceedings of the American Control Conference, pages 1859-1864 vol. 3.

Stramigioli, S., Secchi, C., van der Schaft, A. J., and Fantuzzi, C. (2005). Sampled data systems passivity and discrete port-hamiltonian systems. IEEE Transactions on Robotics, 21(4):574 - 587.

van der Schaft, A. (1999). L2-Gain and Passivity in Nonlinear Control. Springer-Verlag New York, Inc., Secaucus, NJ, USA.

Wang, W. and Slotine, J.-J. (2006). Contraction analysis of time-delayed communications and group cooperation. IEEE Transactions on Automatic Control, 51(4):712717.

Zames, G. (1966). On the input-output stability of timevarying nonlinear feedback systems part one: Conditions derived using concepts of loop gain, conicity, and positivity. IEEE Transactions on Automatic Control, 11(2):228-238. 